\title{
Temperature and pressure behavior of the emission bands from Mn-, Cu-, and Eu-doped ZnS nanocrystals
}

\author{
F. H. Su, ${ }^{\text {a) }}$ Z. L. Fang, B. S. Ma, K. Ding, and G. H. Li \\ National Laboratory for Superlattices and Microstructures, Institute of Semiconductors, Chinese Academy \\ of Sciences, P.O. Box 912, Beijing 100083, China \\ S. J. Xu \\ Department of Physics and HKU-CAS Joint Laboratory on New Materials, The University of Hong Kong, \\ Pokfulam Road, Hong Kong, China
}

(Received 30 October 2003; accepted 5 January 2004)

\begin{abstract}
The temperature and pressure dependence of the photoluminescence from $\mathrm{ZnS}: \mathrm{Mn}^{2+}, \mathrm{ZnS}: \mathrm{Cu}^{2+}$, and $\mathrm{ZnS}: \mathrm{Eu}^{2+}$ nanocrystals were investigated in the temperature range from 10 to $300 \mathrm{~K}$ and under hydrostatic pressure up to $6 \mathrm{GPa}$ at room temperature. The orange emission $(590 \mathrm{~nm})$ from the ${ }^{4} T_{1-}{ }^{6} A_{1}$ transition of $\mathrm{Mn}^{2+}$ ions, the green emission (518 nm) from the $4 f^{6} 5 d^{1}-4 f^{7}$ transition of $\mathrm{Eu}^{2+}$ ions and the blue emission $(460 \mathrm{~nm})$ related to the transition from the conduction band of $\mathrm{ZnS}$ to the $t_{2}$ level of $\mathrm{Cu}^{2+}$ ions were observed in the $\mathrm{Mn}-, \mathrm{Eu}-$, and $\mathrm{Cu}$-doped samples, respectively. It was found that all of these emission bands decrease in intensity with increasing temperature. Among them the intensity of the Mn-orange emission dropped faster. The activation energies were estimated to be 58, 16, and $42 \mathrm{meV}$ for the $\mathrm{Mn}$-orange, Eu-green, and $\mathrm{Cu}$-blue emissions, respectively. A negative pressure coefficient of $-26 \mathrm{meV} / \mathrm{GPa}$ was obtained for the Mn-orange emission, which agrees with the value calculated from the crystal field theory. The pressure coefficient of the $\mathrm{Cu}$-blue emission was found to be $62 \mathrm{meV} / \mathrm{GPa}$, which is almost same as the value of the band gap of bulk $\mathrm{ZnS}$. However, the pressure coefficient of the Eu-green emission is $23 \mathrm{meV} / \mathrm{GPa}$, which is contrary to the predication by the crystal field theory. The strong interaction between the $4 f^{6} 5 d^{1}$ state of the $\mathrm{Eu}^{2+}$ ions and the conduction band of $\mathrm{ZnS}$ may be the origin for the positive pressure coefficient and the small thermal activation energy of Eu-green emission. (C) 2004 American Institute of Physics. [DOI: 10.1063/1.1650894]
\end{abstract}

\section{INTRODUCTION}

Semiconductor nanocrystals (NCs) have attracted much attention in recent years. Because of quantum confinement effect in NCs, semiconductor NCs exhibit unique optical and electronical properties. ${ }^{1}$ However, the surface-related nonradiative recombination may reduce the luminescence efficiency of NCs significantly, thus limiting the applications of semiconductor NCs in optoelectronics. In 1994, Bhargava et al. reported the high luminescence efficiency and short emission lifetimes of $\mathrm{ZnS}$ nanoparticles when $\mathrm{Mn}$ ions were incorporated into the particles. ${ }^{2}$ Since then, there has been much effort devoted to the investigation of semiconductor NCs doped with various impurities. ${ }^{3-5}$ In particular, different impurities-activated ZnS NCs such as ZnS:Mn, CdS:Mn, Zn$\mathrm{S}: \mathrm{Eu}$, and $\mathrm{ZnS}: \mathrm{Cu}$, etc., have been extensively studied. These studies include preparation methods of the materials, ${ }^{5,6}$ structure analysis, ${ }^{7,8}$ and optical properties. ${ }^{9,10}$

An orange emission has been observed in the $\mathrm{ZnS}: \mathrm{Mn}$ NCs, which is attributed to the ${ }^{4} T_{1^{-}}{ }^{6} A_{1}$ transition of $\mathrm{Mn}^{2+}$ ions $^{2}$ in the $\mathrm{ZnS}$ host. However, the similar intraions transition of $\mathrm{Eu}^{2+}$ cannot be observed in bulk $\mathrm{ZnS}$ :Eu because the excited state of $\mathrm{Eu}^{2+}$ are higher or degenerate with the host conduction band. ${ }^{11}$ In contrast to the case of bulk $\mathrm{ZnS}: \mathrm{Eu}$, the intraions emission from the $\mathrm{Eu}^{2+}$ can be observed in

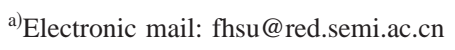

nanocrystals because the band gap of $\mathrm{ZnS}$ NCs becomes wider due to the quantum confinement effect. For example, the intraions transition of $4 f^{6} 5 d^{1}-4 f^{7}$ within $\mathrm{Eu}^{2+}$ ions in Eu-doped ZnS NCs has been observed by several groups. ${ }^{11,12}$ The bulk $\mathrm{ZnS}: \mathrm{Cu}$ has two well-known emission bands, namely, blue and green band. ${ }^{13}$ But for $\mathrm{ZnS}: \mathrm{Cu} \mathrm{NC}$, its luminescence property is still an open problem. Khosravi et al. ${ }^{14}$ observed an emission band peaked at $480 \mathrm{~nm}$, and attributed it to the transition between defect state related to anion vacancies and the copper induced $t_{2}$ level. Bol et al. ${ }^{15}$ reported the observation of green $(470 \mathrm{~nm})$ and red $(600 \mathrm{~nm})$ emissions, which are assigned to the recombination from a shallowly trapped electron to the $t_{2}$ state of $\mathrm{Cu}^{2+}$ ions and the deep localized donor state related to $S^{2-}$ ions, respectively. On the other hand, the emission bands observed by $\mathrm{Xu}$ et al. ${ }^{6}$ consist of two emission peaks centered at 460 and 507 $\mathrm{nm}$. They attributed the two emission bands to the transition from the conduction band and shallow donor state of $\mathrm{ZnS}$ host to the $t_{2}$ state of copper, respectively. In order to extend practical applications of the $\mathrm{ZnS} \mathrm{NCs,} \mathrm{it} \mathrm{is} \mathrm{highly} \mathrm{desirable}$ to have a better understanding of the nature of the impurity related transitions in the NCs.

The temperature- and pressure-dependent photoluminescence (PL) techniques are frequently used to investigate the properties of the luminescence centers in semiconductors. Since externally applied hydrostatic pressure can short the interatomic distance, and thus change the overlap among ad- 
jacent electronic orbits, the pressure dependence of luminescence can provide some useful information about the electronic states of the emitters and the interactions between the luminescence centers and their hosts. Because the temperature influences the crystal fields surrounding luminescence centers and electron-phonon coupling, the different luminescence centers are expected to exhibit different temperature behavior. The temperature and pressure behavior for the orange emission in $\mathrm{ZnS}: \mathrm{Mn} \mathrm{NC}$ were investigated in detail. ${ }^{16-18}$ However, there are relatively few studies on the temperature and pressure behavior for $\mathrm{ZnS}: \mathrm{Cu}$ and $\mathrm{ZnS}: \mathrm{Eu}$ NC. In this article, we report a comparative study on the luminescence spectra from ZnS:Mn, ZnS: $\mathrm{Cu}, \mathrm{ZnS}: \mathrm{Eu} \mathrm{NCs}$ under different temperatures and pressures. The temperature dependence of the emission intensity and the peak energy, the pressure dependence of the peak position for the characteristic emission bands related to the three kinds of doped impurities in ZnS NCs were investigated in detail.

\section{EXPERIMENT}

The samples of $\mathrm{Cu}-$, Eu-, and $\mathrm{Mn}$-doped $\mathrm{ZnS}$ NCs were synthesized with microemulsion and hydrothermal treatment. The detailed preparation process has been reported previously. ${ }^{6}$ The particle size of doped $\mathrm{ZnS}$ NC was determined to be distributed from $3-18 \mathrm{~nm}$ by TEM observation. ${ }^{6}$

The variable-temperature PL measurements were performed by fixing samples on the cold finger of a closed-cycle refrigeration system. The temperature could be varied from 10 to $300 \mathrm{~K}$. The PL measurements under hydrostatic pressure were done in a gasketed diamond-anvil cell (DAC) at room temperature. Some powders of the samples, together with a piece of ruby chip, were placed in a stainless-steel gasket with a hole of $300 \mu \mathrm{m}$ in diameter. The 4:1 methanalethanal mixture was used as pressure-transmitting medium. The pressure was determined by using the standard rubyfluorescence technique and could be varied from 0 to $6 \mathrm{GPa}$.

The $325 \mathrm{~nm}$ line of a He-Cd laser was used as the excitation source. The emitted light was dispersed by a JYHRD1 double grating monochromator and detected by a cooled GaAs photomultiplier operating in the photoncounting mode.

\section{RESULTS AND DISCUSSION}

\section{A. Temperature dependence}

Figure 1 shows the PL spectra measured at 300 and $10 \mathrm{~K}$ for three kinds of $\mathrm{ZnS}$ NCs doped with different dopants. The room-temperature PL spectra from the same samples have been reported elsewhere. ${ }^{6}$ The PL spectrum of $\mathrm{ZnS}: \mathrm{Mn}$ $\mathrm{NCs}$ at $300 \mathrm{~K}$ consists of only one peak at $2.05 \mathrm{eV}$, which is the characteristic emission of $\mathrm{Mn}^{2+}$ ions $\left({ }^{4} T_{1^{-}}{ }^{6} A_{1}\right)$ in $\mathrm{ZnS}$ lattice. $^{2}$ This peak is referred to as Mn-orange emission in the present article. The $\mathrm{Cu}$-doped $\mathrm{ZnS} \mathrm{NCs}$ at room temperature also had an emission peak around $2.7 \mathrm{eV}(460 \mathrm{~nm})$, named as $\mathrm{Cu}$-blue emission henceforth, which is attributed to the transition between conduction band of $\mathrm{ZnS} \mathrm{NC}$ and acceptor-like the $t_{2}\left(d^{9}\right)$ state of $\mathrm{Cu}^{6}{ }^{6}$ It should be noted that the low-energy shoulder at $508 \mathrm{~nm}$ reported previously ${ }^{6}$ is almost invisible at room temperature in this measurement.

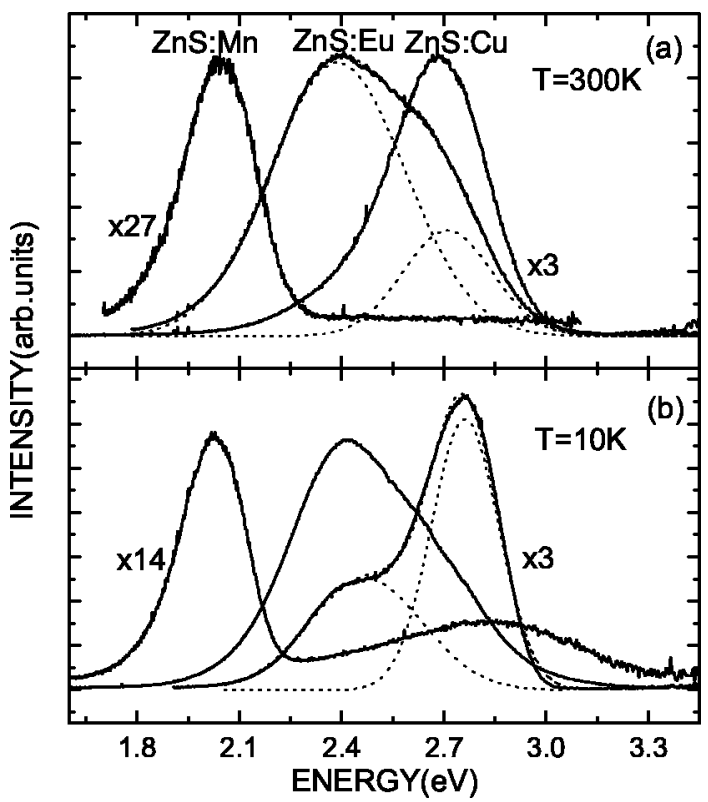

FIG. 1. The PL spectra from $\mathrm{ZnS}: \mathrm{Mn}, \mathrm{ZnS}: \mathrm{Eu}$, and $\mathrm{ZnS}: \mathrm{Cu}$ nanocrystals at 300 and $10 \mathrm{~K}$. The dotted lines are the least-squares fitting curves using two Gaussian functions.

The shoulder becomes visible only in the low-temperature spectra, as shown in Fig. 1. It may be due to the different excitation power in the two measurements. The Eu-doped ZnS NCs have a relatively broad emission band that is similar to the result reported in the reference. ${ }^{6}$ However, the spectrum obtained in the present study seems to consist of two emission bands, which can be resolved into the two Gaussian-like peaks as shown in Fig. 1(a). The main band at $2.4 \mathrm{eV}(518 \mathrm{~nm})$ is assigned to the $\mathrm{Eu}^{2+}$ intraion transition of $4 f^{6} 5 d^{1}-4 f^{7}$ and is referred to as the Eu-green emission. ${ }^{11}$ The high-energy shoulder around $2.7 \mathrm{eV}(460 \mathrm{~nm})$ is tentatively assigned to be a donor-acceptor pair transition in $\mathrm{ZnS}$ host. At low temperatures, a broad emission band around 2.8 $\mathrm{eV}$ was observed in addition to the Mn-orange emission in ZnS:Mn NCs. This band has been discussed in detail and was attributed to the donor-acceptor pair transition related to $\mathrm{ZnS}$ host in Ref. 18. The $10 \mathrm{~K}$ PL spectrum of the Cu-doped $\mathrm{ZnS}$ NCs consists of two emission bands including a main blue emission and a low-energy shoulder at about $2.45 \mathrm{eV}$ $(508 \mathrm{~nm})$. The latter is called the $\mathrm{Cu}$-green emission in the following discussion. The $\mathrm{Cu}$-green emission band has been attributed to the transition from a defect state in $\mathrm{ZnS}$ gap to the $t_{2}$ state of $\mathrm{Cu}^{2+}$ ions. ${ }^{6,19}$ From the low-temperature PL spectra of $\mathrm{ZnS}: \mathrm{Eu}$, it can be seen that the relative intensity of the blue emission band with respect to that of the Eu-green peak becomes even smaller.

The more PL spectra for the three samples under different temperatures are shown in Fig. 2. As can be seen in Fig. 2(a), the blue emission of the Mn-doped ZnS NCs quenches faster than their orange emission with increasing temperature. At room temperature, the blue emission quenches completely while the orange emission is still observable. In contrast to the case of the Mn-doped ZnS NCs, the high-energy peaks of the $\mathrm{Eu}-$ and $\mathrm{Cu}$-doped $\mathrm{ZnS}$ NCs quench slower than their corresponding partners at the lower energy side. For 


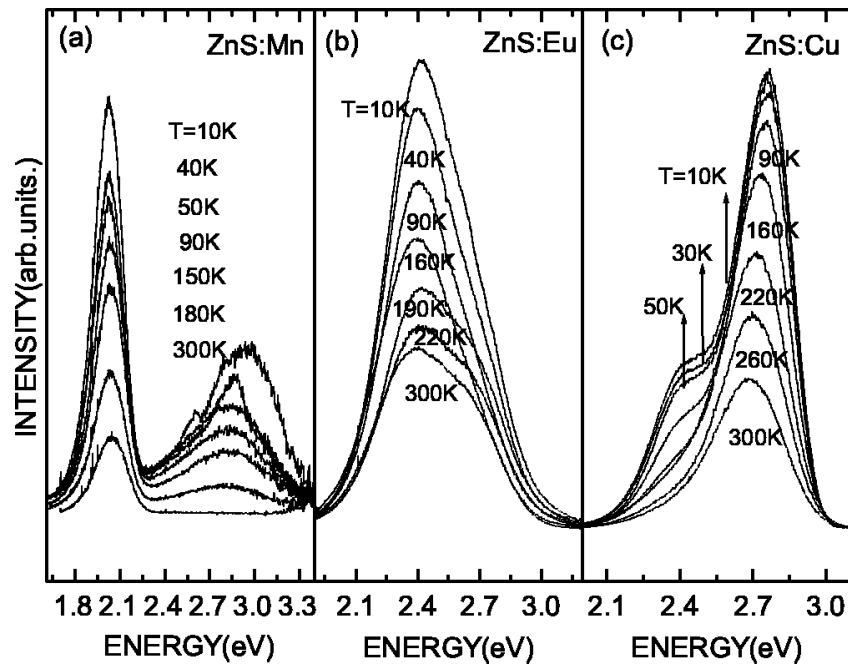

FIG. 2. The PL spectra for $\mathrm{ZnS}: \mathrm{Mn}, \mathrm{ZnS}: \mathrm{Eu}$, and $\mathrm{ZnS}: \mathrm{Cu}$ nanocrystals at various temperatures.

example, the low-energy emission shoulder of the $\mathrm{ZnS}: \mathrm{Cu}$ $\mathrm{NCs}$ becomes unresolved when the temperature is above around $220 \mathrm{~K}$.

It is worth mentioning that the major emission band of the Mn-doped ZnS NCs has a different quenching rate from those of the Eu-and Cu-doped $\mathrm{ZnS}$ NCs. For example, when the temperature is increased from $10 \mathrm{~K}$ to room temperature, the intensity of Mn-orange emission drops to only $20 \%$ of its maximum value at $10 \mathrm{~K}$. However, the intensity of $\mathrm{Cu}$-blue and Eu-green emissions decreases to 33 and $40 \%$ of their maxima at $10 \mathrm{~K}$, respectively. The integrated intensity of the $\mathrm{Mn}$-orange, Eu-green, and $\mathrm{Cu}$-blue emissions are shown in Fig. 3 as a function of temperature. For the $\mathrm{Eu}-$ and $\mathrm{Cu}-$ doped $\mathrm{ZnS} \mathrm{NCs,} \mathrm{the} \mathrm{temperature} \mathrm{dependence} \mathrm{of} \mathrm{the} \mathrm{intensity}$ of the major emission bands can be well described by a thermal quenching theory ${ }^{20}$

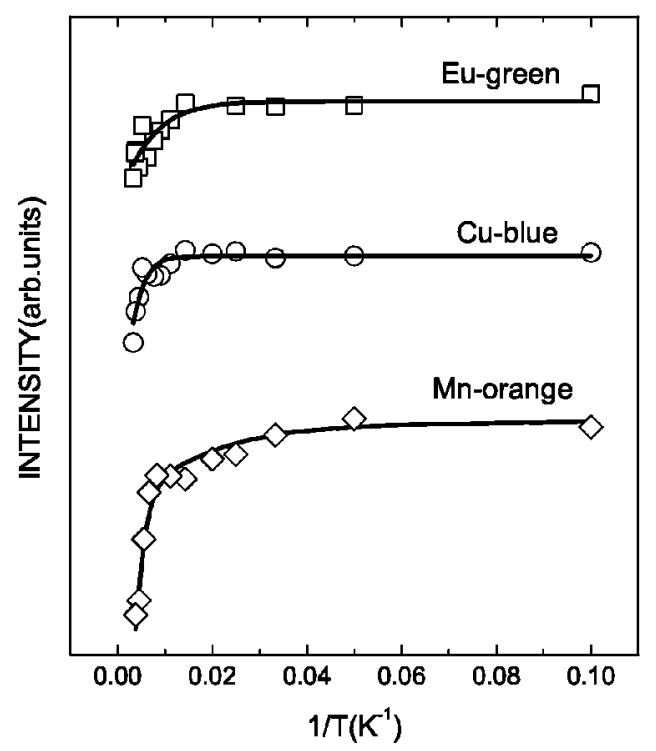

FIG. 3. Temperature dependence of the integrated intensity for the emission bands from $\mathrm{ZnS}: \mathrm{Mn}, \mathrm{ZnS}: \mathrm{Eu}$, and $\mathrm{ZnS}: \mathrm{Cu}$ nanocrystals. The cure-fit lines through Eu-green and Cu-blue were obtained using Eq. (1). The solid line through Mn-orange is the fitting results using Eq. (2).

$$
I(T)=\frac{I_{0}}{1+a \exp \left(-E / k_{B} T\right)},
$$

where $E$ is the activation energy for the thermal quenching, $k_{B}$ is the Boltzmann constant, $a$ is a constant, and $I_{0}$ is the emission intensity at $0 \mathrm{~K}$. A fitting result to the experimental data of the two samples using Eq. (1) is presented in Fig. 3 by the solid lines. The obtained activation energy is $16 \pm 3$ $\mathrm{meV}$ for the Eu-green emission, and $42 \pm 8 \mathrm{meV}$ for the $\mathrm{Cu}$ blue emission. However, the temperature dependence of the Mn-orange emission cannot be well simulated by the Eq. (1). According to the experimental data displayed in Fig. 3, there may be two thermal quenching processes for the Mn-orange emission: a slow one during the temperature range from 10 to $90 \mathrm{~K}$ and a fast one for the temperatures above $90 \mathrm{~K}$. Therefore, the variation of the intensity of the Mn-orange emission with temperature could be fitted using the following equation:

$$
I(T)=\frac{I_{0}}{1+a_{1} \exp \left(-E_{1} / k_{B} T\right)+a_{2} \exp \left(-E_{2} / k_{B} T\right)} .
$$

Two thermal activation energies of $E_{1}=7 \pm 2 \mathrm{meV}, E_{2}=58$ $\pm 15 \mathrm{meV}$ are obtained from the least-squares fitting to the experimental data.

For the Mn-orange emission in $\mathrm{ZnS} \mathrm{NCs,} \mathrm{the} \mathrm{optical}$ excitation from the $325 \mathrm{~nm}$ laser is "nondirect." In other words, element excitations are excited in the $\mathrm{ZnS}$ host by the photons, and then parts of them transfer to the Mn ions, followed by a radiative recombination around the Mn ions. This means that two thermal quenching channels may exist for the Mn-orange emission. The thermal quenching process with an activation energy of $58 \mathrm{meV}$ is likely related to the excitons' thermal dissociation in $\mathrm{ZnS}$ host since the thermal activation energy of $58 \mathrm{meV}$ is very close to the binding energy of excitons in $\mathrm{ZnS}: \mathrm{Mn}$ nanoparticles $(60 \mathrm{meV}) .{ }^{21} \mathrm{The}$ other with much smaller thermal activation energy of $7 \mathrm{meV}$ may be associated with the Mn ions themselves, for instance, the thermal desorption of excitons from the Mn ions. We also note that the thermal activation energy of the Eu-green emission band is only $16 \mathrm{meV}$. It may be due to the fact that the $4 f^{6} 5 d$ excited state of the $\mathrm{Eu}^{2+}$ ion is very close to the conduction band edge of $\mathrm{ZnS}$ host. ${ }^{11}$ Besides causing the small thermal activation energy, the strong interaction between, $\mathrm{Eu}^{2+}$ ion and $\mathrm{ZnS}$ host may be responsible for the unusual pressure behavior of the Eu-green emission band, which will be discussed in detail later. In the Cu-doped NCs, the photo-generated electrons in the conduction band of $\mathrm{ZnS}$ host can recombine directly with the holes on the $t_{2}$ states of $\mathrm{Cu}^{2+}$ ions, which produces the $\mathrm{Cu}$-blue emission band. Therefore, the quenching of the $\mathrm{Cu}$-blue emission may result from the thermal escape of the holes in $t_{2}$ levels of $\mathrm{Cu}^{2+}$ ions.

The temperature dependence of the peak energies of the emission bands in the three samples is displayed in Fig. 4. The peak energies were obtained from least-square fitting to the measured PL spectra using two Gaussian line profiles when the spectrum consists of two overlapping emission bands, as shown in Fig. 1. It can be seen from Fig. 4 that the peak positions of the intraion emissions such as the Mn- 


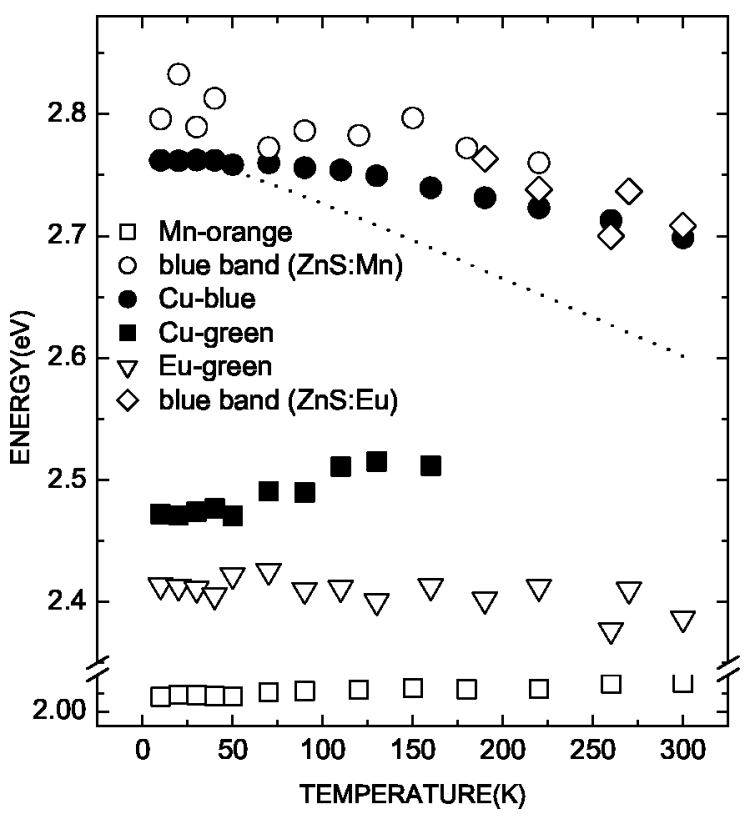

FIG. 4. The peak energies for the emission bands in three samples vs temperature. The dotted line is the temperature dependence for the band gap of bulk $\mathrm{ZnS}$, which is shifted vertically by $1.07 \mathrm{eV}$ for comparison.

orange emission and the Eu-green emission depend weakly on temperature, which is in agreement with those reported in Ref. 18. Both blue bands in $\mathrm{ZnS}: \mathrm{Mn}$ and $\mathrm{ZnS}: \mathrm{Eu}$ samples exhibit a small redshift with increasing temperature. As for $\mathrm{ZnS}: \mathrm{Cu}$ case, the $\mathrm{Cu}$-blue emission shifts toward lower energy when the temperature increases. In contrast with the $\mathrm{Cu}$-blue band, the $\mathrm{Cu}$-green emission shifts toward higher energy with increasing temperature. The temperature dependence of the band gap of bulk $\mathrm{ZnS},{ }^{22}$ which is shifted vertically by $1.07 \mathrm{eV}$ for comparison, is also plotted as a dotted line in Fig. 4. Obviously, the temperature dependence of the peak position of the $\mathrm{Cu}$-blue emission does not follow that of the band gap of bulk ZnS exactly. Compared with the band gap of bulk $\mathrm{ZnS}$, the redshift of the Cu-blue emission with temperature is smaller. It implies that the temperature dependence of the $t_{2}$ level of the $\mathrm{Cu}^{2+}$ ions is very different from that of the valence band since the $\mathrm{Cu}$-blue emission is due to the transition from the conduction band edge of $\mathrm{ZnS}$ host to the $t_{2}$ state of the $\mathrm{Cu}^{2+}$ ions. Unlike the valence band of $\mathrm{ZnS}$ host, the $t_{2}$ state likely moves to a lower energy position in the band gap with increasing temperature. The observed blueshift of the $\mathrm{Cu}$-green emission, which is supposed to be a transition from a defect level of $\mathrm{ZnS}$ host to the $t_{2}$ states of $\mathrm{Cu}^{2+}$ ions, also supports the above suggestion if the variation with temperature of the defect level is smaller than that of the conduction band due to its localized character.

\section{B. Pressure dependence}

Figure 5 depicts the normalized PL spectra of Mn-, Eu-, and $\mathrm{Cu}$-doped $\mathrm{ZnS}$ NCs measured at room temperature as a function of pressure. The dip superimposed on the PL spectra of $\mathrm{ZnS}: \mathrm{Cu}$ under the pressure value above 4.0 GPa comes from the absorption by a defect state of the front diamond equipped in the DAC. It can be confirmed by the absorption

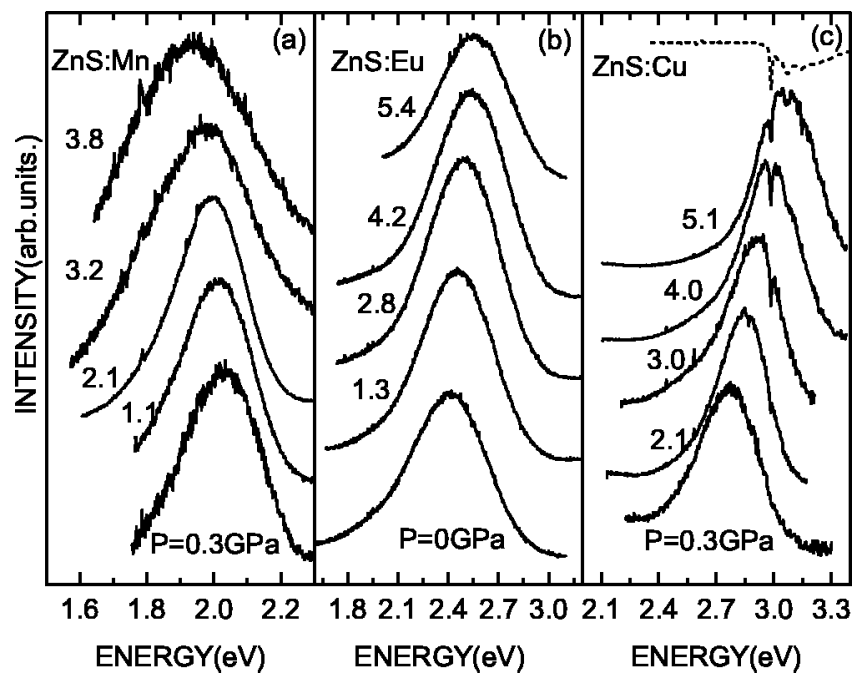

FIG. 5. Normalized PL spectra under different pressures for ZnS:Mn, Zn$\mathrm{S}: \mathrm{Eu}$, and $\mathrm{ZnS}: \mathrm{Cu}$ nanocrystals. The dashed line in (c) is the absorption spectrum of diamond.

spectrum (dashed line) of the diamond shown in Fig. 5(c). It should be noted that the only the Eu-green emission band can be observed in $\mathrm{ZnS}$ :Eu loaded inside DAC, as shown in Fig. 5(b). The blue emission, which can be observed as a higher energy shoulder in usual PL measurements, becomes invisible in the pressure-dependent PL experiment. It may be due to the weaker excitation power in the pressure measurements.

It is clear that the Mn-orange emission shifts to lower energy positions with increasing pressure while both the Eugreen and $\mathrm{Cu}$-blue emission exhibit a blueshift. The shift of emission energies of various emission bands with pressure can be seen more clearly in Fig. 6 in which the pressure dependence of the peak energy for the Mn-orange, Eu-green, and $\mathrm{Cu}$-blue emissions is drawn. The solid lines in Fig. 6 represent the result of the least-squares fitting to the experimental data using a linear relation

$$
E(P)=E_{0}+\alpha P,
$$

where $\alpha$ is the pressure coefficient, $E_{0}$ represents the emission energy at $P=0 \mathrm{GPa}$. The obtained pressure coefficients for the main emission bands in the $\mathrm{Mn}-$, Eu-, and $\mathrm{Cu}$-doped $\mathrm{ZnS} \mathrm{NCs}$ are $-26 \pm 5,23 \pm 2$, and $62 \pm 3 \mathrm{meV} / \mathrm{GPa}$, respectively.

The pressure coefficient of $\mathrm{Mn}^{2+}$ emission (the $\mathrm{Mn}$ orange emission in the present work) in $\mathrm{ZnS}: \mathrm{Mn}$ can be calculated by using the crystal field theory. ${ }^{23}$ According to the theory, the energy level of $\mathrm{Mn}^{2+}$ ions in $\mathrm{ZnS}: \mathrm{Mn}$ can be expressed as a function of the crystal field parameter $D q$ and the Racah parameters $B$ and $C$. Therefore, the pressure coefficient of the peak energy of the orange emission $d E / d P$ can be expressed as a function of $d D q / d P$ and $d B / d P$, assuming that the Racah parameter ratio $C / B$ is independent on the pressure, ${ }^{24}$

$$
\frac{d E}{d P}=\delta \frac{d D q}{d P}+\left(E_{0}-D q \delta\right) \frac{1}{B} \frac{d B}{d P},
$$




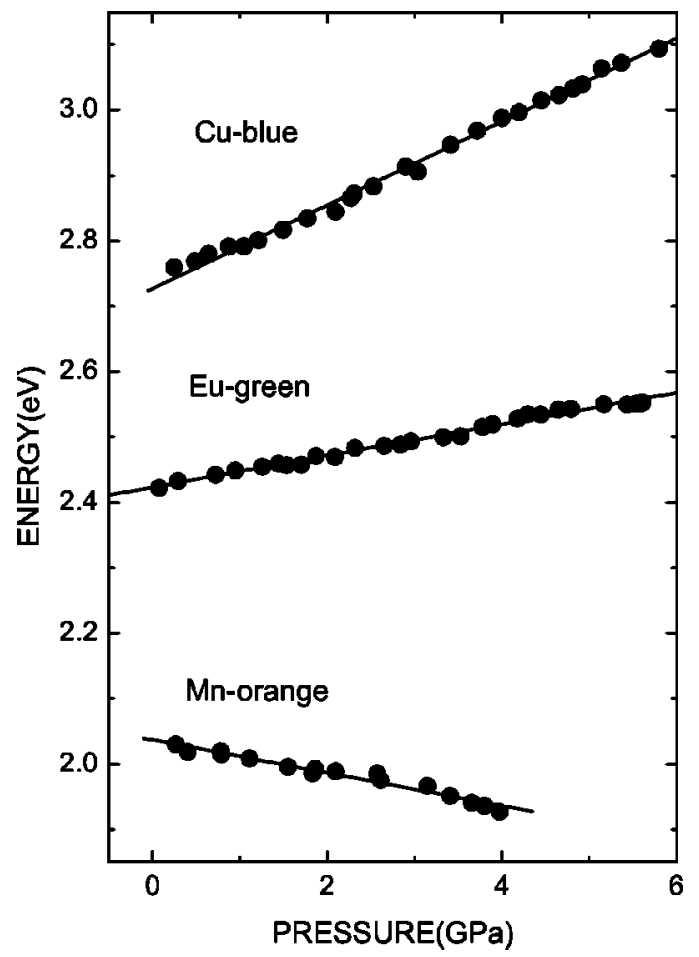

FIG. 6. Pressure dependence of the peak energies of three characteristic emissions for $\mathrm{ZnS}: \mathrm{Mn}, \mathrm{ZnS}: \mathrm{Eu}$, and $\mathrm{ZnS}: \mathrm{Cu}$ nanocrystals. The solid lines are the least-squares fitting curves to the data.

where $\delta=d(E / B) / d(D q / B)$ is the slope of the curve $E / B$ versus $D q / B$ at the value of $D q / B$ derived from the corresponding transition ${ }^{4} T_{1}$ to ${ }^{6} A_{1}$ in the Sugano-Tanabe diagram. The value of $\delta=-10$ for the $\mathrm{Mn}^{2+}$ ions is adopted here. In a point ion model, the $D q$ is proportional to $R^{-5}$, where $R$ represents the distance between the impurity ion and surrounding sulphur ions. ${ }^{24}$ Therefore $d D q / d P$ can be expressed as

$$
\frac{d D q}{d P}=\frac{5}{3} k D q,
$$

where $k=-1 / V d V / d P=1.27 \times 10^{-2} \mathrm{GPa}^{-1}$ (Ref. 25) is the volume compressibility of $\mathrm{ZnS} . E_{0}$ is the PL peak energy of the Mn-orange emission at $P=0 \mathrm{GPa}$. The parameters of $D q$ and $B$ vary from 512 to $519 \mathrm{~cm}^{-1}$ and from 490 to $520 \mathrm{~cm}^{-1}$ for 3-10 nm sized ZnS:Mn NCs, ${ }^{26}$ respectively. The calculated pressure coefficient of the Mn-orange emission from Eq. (5) is about $-30 \mathrm{meV} / \mathrm{GPa}$, which agrees well with the experimental value $(-26 \pm 5 \mathrm{meV} / \mathrm{GPa})$.

For the $\mathrm{Cu}$-doped $\mathrm{ZnS} \mathrm{NC}$, the $\mathrm{Cu}$-blue emission originates from the transition from the conduction band of $\mathrm{ZnS}$ host to the $t_{2}$ level of $\mathrm{Cu}^{2+}$ ions. The experimental value of the pressure coefficient is $62 \pm 3 \mathrm{meV} / \mathrm{GPa}$, which is very close to that of the band gap of bulk $\mathrm{ZnS}[63.5 \mathrm{meV} / \mathrm{GPa}$ (Ref. 27)]. It indicates that the variation of the $t_{2}$ energy level with pressure follows the valence band edge of $\mathrm{ZnS}$ host if we assume that the pressure coefficient of the $\mathrm{ZnS}$ nanoparticles is equal to that of the bulk $\mathrm{ZnS}$. In other words, the energy difference between the $t_{2}$ level and the valence band edge of $\mathrm{ZnS}$ host is pressure independent. It has been demonstrated that the pressure behavior of the band to a shallow acceptor transition is similar to that of the band gap in general. ${ }^{28}$ Our results support such a conclusion in literature and also indicate that the $t_{2}$ level of the $\mathrm{Cu}^{2+}$ ions behaves like a shallow acceptor level under the condition of pressure externally applied, although it comes from the $d$ electron state of the $\mathrm{Cu}$ ions. According to the deformation calculation, the valence band edge of bulk $\mathrm{ZnS}$ has a blueshift as the pressure increases. ${ }^{29}$ On the other hand, the crystal field theory predicts that externally applied pressure will raise the $t_{2}$ level of $\mathrm{Cu}^{2+}$ ions in energy. In the cubic crystal field, the $d$ electron state of the $\mathrm{Cu}^{2+}$ ion splits into two levels, i.e., triply degenerate state $t_{2}$ and doubly degenerate state $e_{g}$. The $t_{2}$ is higher than the original $d$-level by $4 D q$, and the $e_{g}$ lower by $6 D q$. The distance of $\mathrm{Cu}-\mathrm{S}$ is shortened by the pressure and then the value of $D q$ will increase. Therefore, the $t_{2}$ level of $\mathrm{Cu}^{2+}$ ions has a blueshift with increasing pressure. If the pressure-induced blueshifts of the valence band edge and the $t_{2}$ state are the same, the pressure coefficients for the $\mathrm{Cu}$-blue emission and $\mathrm{ZnS}$ band gap should be the same too. Indeed, our pressure experiment demonstrates this point. It is worth indicating that the temperature dependence of the peak energy for the $\mathrm{Cu}$-blue emission seems not to support the conclusion. In contrast with the pressuredependent experiment, the temperature-dependent experiment suggests that the variation of the $t_{2}$ level with temperature does not follow that of the valence band edge. The origin for the disagreement between these two behaviors is unknown yet.

The ground state of the free $\mathrm{Eu}^{2+}$ ion is ${ }^{8} S_{7 / 2}$ with $4 f^{7}$ configuration, and its lowest lying excited configuration is $4 f^{6} 5 d^{1}$. For the $\mathrm{Eu}^{2+}$ ion incorporated in $\mathrm{ZnS}$ host, the $4 f^{6} 5 d^{1}$ excited state splits into $E_{g}$ and $T_{2 g}$ states by the crystal field of the host. The Eu-green emission observed in the present work is attributed to the $\mathrm{Eu}^{2+}$ intraion transition of $4 f^{6} 5 d^{1}\left(T_{2 g}\right)-4 f^{7}$. The change of the crystal field surrounding the $\mathrm{Eu}^{2+}$ under action of the external pressure is responsible to the pressure-induced shift of the Eu-green emission. The dependence of the $4 f^{6} 5 d^{1}\left(T_{2 g}\right)-4 f^{7}$ transition energy on the crystal field strength $D q$ is described as ${ }^{30}$

$$
E_{f-d}=3.3-0.41 \times(10 D q) .
$$

From the crystal field theory, when an external pressure is applied, the increase of the crystal field strength is expected because of the compression in bond length. Therefore, the $4 f^{6} 5 d^{1}\left(T_{2 g}\right)-4 f^{7}$ transition of $\mathrm{Eu}^{2+}$ in $\mathrm{ZnS}$ host should have a redshift with increasing pressure according to Eq. (8). In other words, a negative pressure coefficient of the $4 f^{6} 5 d^{1}\left(T_{2 g}\right)-4 f^{7}$ transition of $\mathrm{Eu}^{2+}$ in $\mathrm{ZnS}$ host is expected by the crystal field theory. In fact, it has been experimentally observed by people in several types of Eu-doped bulk materials such as $\mathrm{CaS}: \mathrm{Eu}$ and $\mathrm{BaBr}_{2}$ :Eu. ${ }^{31,32}$ However, the pressure coefficient of the Eu-green emission measured in the present work is about $20 \mathrm{meV} / \mathrm{GPa}$, which is in contrast to the predication by the crystal field theory.

In bulk $\mathrm{ZnS}: \mathrm{Eu}$, the $T_{2 g}$ state is located about $0.35 \mathrm{eV}$ above the edge of conduction band of the host. Therefore, no intraion $d-f$ transition was observed. ${ }^{11}$ However, in $\mathrm{ZnS}: \mathrm{Eu}$ $\mathrm{NC}$, the situation may be quite different. The conduction band edge of $\mathrm{ZnS}$ host definitely goes up in energy due to the 
strong quantum size effect. This may enable the conduction band edge to be above the excited state of $\mathrm{Eu}^{2+}$. The intraion transition may thus be observed. Chen et al. ${ }^{11}$ has demonstrated that in order to observe the intraion transition, the diameter of a $\mathrm{ZnS}$ nanoparticle should be at least smaller than $3.5 \mathrm{~nm}$. As mentioned earlier, the particle diameters of the samples studied in the present work are distributed from 3 to $18 \mathrm{~nm}$. We thus believe that some $\mathrm{ZnS}$ :Eu particles with smaller diameters satisfy the condition given by $\mathrm{Chen}^{11}$ or approach the condition. For these $\mathrm{ZnS}$ particles, their conduction-band edge resonances with or fairly close to the $T_{2 g}$ excited state of $\mathrm{Eu}^{2+}$ ions. A strong coupling between the electrons in the conduction band of $\mathrm{ZnS}$ host and $d$ electrons in $\mathrm{Eu}$ ions is thus expected, ${ }^{11}$ which may cause the unusual observed pressure behavior of the Eu-green emission in ZnS:Eu NCs. The obtained small thermal activation energy for the Eu-green emission in the study of temperature dependence of the emission intensity may also come from the same cause. More theoretical and experimental work should be done for further understanding the role of the $4 f^{6} 5 d^{1}$ electrons of Eu ions in optical properties of $\mathrm{ZnS}: \mathrm{Eu}$ NCs.

\section{CONCLUSION}

In conclusion, we have investigated the temperature and pressure dependence of PL for $\mathrm{Mn}$-, Eu-, and $\mathrm{Cu}$-doped $\mathrm{ZnS}$ NCs. The characteristic emission bands related to the three kinds of doped impurities, namely, the Mn-orange $(2.05 \mathrm{eV})$, Eu-green $(2.4 \mathrm{eV})$, and $\mathrm{Cu}-$ blue $(2.7 \mathrm{eV})$ emission, are addressed in the present study. The thermal quenching behavior of the three characteristic emissions is found to be quite different, which reflects the nature of their different transition mechanisms. The energies of the Mn-orange and Eu-green emissions are weakly dependent on the temperature, which is the character of the intraion transitions of doped ions. The $\mathrm{Cu}$-blue emission exhibits temperature and pressure behavior like a transition from the conduction band to a shallow acceptor level. The pressure coefficient of the Mn-orange emission is well consistent with the value calculated using the crystal field theory. The pressure coefficient of the $\mathrm{Cu}$-blue emission is very close to that of the band gap of bulk $\mathrm{ZnS}$. However, the experimental pressure coefficient of the Eugreen emission is found to conflict with the value predicated by the crystal field theory. The strong interaction between the excited state of $\mathrm{Eu}^{2+}$ ions and the conduction band of $\mathrm{ZnS}$ is supposed to be responsible for the positive pressure coefficient and the small thermal activation energy of the Eu-green emission.

\section{ACKNOWLEDGMENTS}

This work was partly supported by the National Natural Science Foundation of China (Contract No. 60076012) and the special funds for Major State Basic Research Project of China (No. G2001CB3095). The work in Hong Kong was supported by the HKU URC Grant (No. 10204008).

${ }^{1}$ Y. Wang and N. Herron, J. Phys. Chem. 95, 525 (1991), and references cited therein.

${ }^{2}$ R. N. Bhargava, D. Gallagher, X. Hong, and A. Nurmikko, Phys. Rev. Lett. 72, 416 (1994).

${ }^{3}$ G. Counio, S. Esnouf, and J.-P. Boilot, J. Phys. Chem. 100, 20021 (1996).

${ }^{4}$ A. A. Khosravi, M. Kundu, L. Jatwa, S. K. Deshpande, U. A. Bhagwat, and S. K. Kulkarni, Appl. Phys. Lett. 67, 2702 (1995).

${ }^{5}$ W. Chen, R. Sammynaiken, and Y. Huang, J. Appl. Phys. 88, 5188 (2000).

${ }^{6}$ S. J. Xu, S. J. Chua, B. Liu, L. M. Gan, C. H. Chew, and G. Q. Xu, Appl. Phys. Lett. 73, 478 (1998).

${ }^{7}$ T. A. Kennedy, E. R. Glaser, P. B. Klein, and R. N. Bhargava, Phys. Rev. B 52, R14 356 (1995).

${ }^{8}$ Y. L. Soo, Z. H. Ming, S. W. Huang, Y. H. Kao, R. N. Bhargava, and D. Gallagher, Phys. Rev. B 50, 7603 (1994).

${ }^{9}$ I. Yu, T. Isobe, and M. Senna, J. Phys. Chem. Solids 57, 373 (1996).

${ }^{10}$ M. A. Chamarro, V. Voliotis, R. Grousson, P. L. Lavallard, T. Gacoin, G. Counio, J. P. Boilot, and R. Cases, J. Cryst. Growth 159, 853 (1996).

${ }^{11}$ W. Chen, J.-O. Malm, V. Zwiller, Y. Huang, S. Liu, R. Wallenberg, J.-O. Bovin, and L. Samuelson, Phys. Rev. B 61, 11021 (2000).

${ }^{12}$ S. M. Liu, H. Q. Guo, Z. H. Zhang, F. Q. Liu, and Z. G. Wang, Chin. Phys. Lett. 17, 609 (2000).

${ }^{13}$ A. Suzuki and S. Shionoya, J. Phys. Soc. Jpn. 31, 1455 (1971).

${ }^{14}$ A. A. Khosravi, M. Kundu, L. Jatwa, S. K. Deshpande, U. A. Bhagwat, M. Sastry, and S. K. Kulkarni, Appl. Phys. Lett. 67, 2702 (1995).

${ }^{15}$ A. A. Bol, J. Ferwerda, J. A. Bergwerff, and A. Meijerink, J. Lumin. 99, 325 (2002).

${ }^{16}$ W. Chen, G. Li, J.-O. Malm, Y. Huang, and R. Wallenberg, J. Lumin. 91, 139 (2000).

${ }^{17}$ W. Chen, F. H. Su, G. H. Li, A. G. Joly, J.-O. Malm, and J.-O. Bovin, J. Appl. Phys. 92, 1 (2002).

${ }^{18}$ F. H. Su, B. S. Ma, Z. L. Fang, K. Ding, G. H. Li, and W. Chen, J. Phys.: Condens. Matter 14, 12657 (2002).

${ }^{19}$ L. Sun, C. Liu, C. Liao, and C. Yan, Solid State Commun. 111, 483 (1999).

${ }^{20}$ K. Kosai, B. T. Fitzpatrick, H. G. Grimmeiss, R. N. Bhargava, and G. F. Neumak, Appl. Phys. Lett. 35, 194 (1979).

${ }^{21}$ M. Tanaka and Y. Masumoto, Chem. Phys. Lett. 324, 249 (2000).

${ }^{22}$ Y. F. Tsay, S. S. Mitra, and J. F. Vetelino, J. Phys. Chem. Solids 34, 2167 (1973).

${ }^{23}$ G. L. House and H. G. Drickamer, J. Chem. Phys. 67, 3230 (1977).

${ }^{24}$ S. Ves, K. Strossner, W. Gebhardt, and M. Cardona, Phys. Rev. B 33, 4077 (1986).

${ }^{25}$ R. A. Montalva and D. W. Langer, J. Appl. Phys. 41, 4101 (1970).

${ }^{26}$ W. Chen, R. Sammynaiken, Y. Huang, J.-O. Malm, R. Wallenberg, J.-O. Bovin, V. Zwiller, and N. A. Kotov, J. Appl. Phys. 89, 1120 (2001).

${ }^{27}$ S. Ves, U. Schwarz, N. E. Christensen, K. Syassen, and M. Cardona, Phys. Rev. B 42, 9113 (1990)

${ }^{28}$ D. J. Wolford and J. A. Bradley, Solid State Commun. 53, 1069 (1985).

${ }^{29}$ S.-H. Wei and A. Zunger, Phys. Rev. B 60, 5404 (1999).

${ }^{30}$ W. Chen, J.-O. Malm, V. Zwiller, R. Wallenberg, and J.-O. Bovin, J. Appl. Phys. 89, 2671 (2001).

${ }^{31}$ C. Chen, K. L. Teo, T. C. Chong, Y. H. Wu, T. S. Low, R. Carey, D. M. Newman, I. Viney, J. P. Wu, and J. Bickerton, J. Appl. Phys. 93, 2559 (2003).

${ }^{32}$ T. Tröster, S. Schweizer, M. Secu, and J.-M. Spaeth, J. Lumin. 99, 343 (2002) 